\title{
Controlled-Source Magnetotellurics: Source Effects
}

\author{
Torquil Smith and $\mathrm{Ki} \mathrm{Ha} \mathrm{Lee}$
}

\author{
Earth Sciences Division \\ Ernest Orlando Lawrence Berkeley National Laboratory \\ University of California \\ Berkeley, California 94720
}

April 1999

This work was supported by Assistant Secretary for Energy Efficiency and Renewable Energy, Geothermal Division, of the U.S. Department of Energy under Contract No. DE-AC03-76SF00098. 


\section{DISCLAIMER}

This report was prepared as an account of work sponsored by an agency of the United States Government. Neither the United States Government nor any agency thereof, nor any of their employees, make any warranty, express or implied, or assumes any legal liability or responsibility for the accuracy, completeness, or usefulness of any information, apparatus, product, or process disclosed, or represents that its use would not infringe privately owned rights. Reference herein to any specific commercial product, process, or service by trade name, trademark, manufacturer, or otherwise does not necessarily constitute or imply its endorsement, recommendation, or favoring by the United States Government or any agency thereof. The views and opinions of authors expressed herein do not necessarily state or reflect those of the United States Government or any agency thereof. 


\section{DISCLAIMER}

Portions of this document may be illegible in electronic image products. Images are produced from the best available original document. 


\section{Controlled-Source Magnetotellurics: Source Effects}

\section{Introduction}

Natural source and controlled source magnetotellurics (MT) are both used to obtain an image of earth conductivity but the techniques differ in the nature of the source fields that induce currents in the earth. In natural source MT, naturally occurring ultralow frequency variations in the earth's magnetic field are used, whereas in controlled source MT (CSMT) these are augmented with man-made fields to increase the available signal strength and bandwidth. The standard MT response functions are given by ratios of the induced electric field to the magnetic field (impedance) as a function of frequency, converted into apparent resistivity and impedance phase. As frequency decreases, the depth of penetration of fields (skin depth) increases. In natural source MT, the source wavefields are generally well approximated by plane waves, and this property considerably simplifies the computation needed for interpretation. In CSMT, the artificial sources necessarily have finite spatial extent, resulting in a rich wavenumber content when considered in a spatial Fourier domain. At distances more than three to five skin depths from the source, CSMT measurements and natural source MT measurements give essentially identical impedances, simplifying interpretation. Closer to the source, the higher wavenumber components of the fields become important, significantly complicating interpretation. In principle, one could interpret CSMT data in the nearsource region by means of a full electromagnetic (EM) modeling analysis, but this procedure is so computationally difficult that it is done infrequently (Newman, 1995; Xie and Lee, 1995).

In this paper we evaluate the CSMT impedance in the wavenumer domain, and compare the result with the full EM impedance obtained from the exact solution over the layered earth. Preliminary results show that we can find a pair of wavenumbers that reproduces the full EM impedance over the frequency range affected by source effects. This observation suggests that it is now possible to obtain the electrical resistivity using the low-frequency EM impedance data that can be represented by a pair of wavenumbers, thereby greatly simplifying the computational requirements. The process will involve a non-linear inversion of near-source impedance data for a pair of wavenumbers and a layered-earth resistivity structure.

\section{Wavenumber analysis}

Consider induction at horizontal wavenumbers $k_{x}$ and $k_{y}$. One can write a solution in the form

$$
\vec{E}=(\alpha, \beta, \gamma) e^{-i k_{x} x-i k_{y} y-i k_{z} z}
$$

at frequency $\omega$, where $\alpha$ and $\beta$ are constants determining the polarization of the electric field. Substitution of equation (1) into the equations relevant for induction in a homogeneous medium, $\nabla^{2} \vec{E}+i \sigma \omega \mu \vec{E}=0$, yields values for $k_{z}$, 


$$
k_{z}^{2}=i \sigma \omega \mu-k_{x}^{2}-k_{y}^{2},
$$

where $\sigma$ is the conductivity of the medium and $\mu$ is its permeability. An $e^{-i \omega t}$ time dependence is used throughout. Similarly, the divergence-free condition, $\nabla \cdot \vec{E}=0$, applied to equation (1) yields

$$
\gamma=-\frac{\alpha k_{x}+\beta k_{y}}{k_{z}} .
$$

This solution gives an impedance for the $H$-polarization

$$
\frac{E_{x}}{H_{y}}=-\frac{\omega \mu}{k_{z}-\not k_{x} / \alpha} .
$$

For non-zero $k_{x}$ and $k_{y}$, as frequency approaches zero, $k_{z}$ and $\gamma$ become purely imaginary, so the impedance phase goes to $90^{\circ}$. Quantities $k_{z}$ and $\gamma$ go to constant values, so the impedance magnitude decreases as $\omega$ approaches zero.

Assuming a solution of the form for the magnetic field

$$
\vec{H}=(a, b, c) e^{-i k_{x} x-i k_{y} y-i k_{z} z}
$$

one could derive similar results;

$$
\begin{aligned}
& k_{z}^{2}=i \omega \mu \sigma-k_{x}^{2}-k_{y}^{2}, \\
& c=-\frac{a k_{x}+b k_{y}}{k_{z}}
\end{aligned}
$$

and

$$
\frac{E_{x}}{H_{y}}=\frac{i}{\sigma}\left(k_{z}-c k_{y} / b\right) .
$$

For non-zero $k_{x}$ and $k_{y}$, as $\omega \rightarrow 0$, this impedance approaches a real constant, with magnetic fields and electric fields in phase. Evidently, these very simple solutions display the same asymptotic behavior as the more complicated solutions for induction by a horizontal magnetic dipole and by a grounded electric dipole, respectively.

In the far-field regions, CSMT impedance measurements are commonly interpreted assuming a zero wavenumber $\left(k_{x}=k_{y}=0\right)$ source-field approximation. Approaching the near-field region, this approximation breaks down. CSMT impedances can be better approximated assuming non-zero horizontal wavenumbers. The source- 
receiver separation $r$ yields the most obvious scale $1 / r$ for the horizontal wavenumbers $k_{x}$ and $k_{y}$ (unit of the wavenumber is $\mathrm{m}^{-1}$ ). For a given source dipole orientation one expects to use different horizontal wavenumber pairs $k_{x}$ and $k_{y}$ for different receiver locations at the same radius. All examples given below will be for an $x$-oriented electric dipole source $\left(J_{x}\right)$ at the origin, with receiver positions in either a broadside position (on the $y$-axis) or an end-on position (on the $x$-axis). In both positions the horizontal magnetic field is in the $y$ direction only, so we choose $a=0$ in equation (5). Apparent resistivity and phase are plotted for receivers in the broadside (Figures 1 and 2) and endon (Figures 3 and 4) locations. Both values calculated from the full solution for induction in a half-space by a horizontal electric dipole and for single horizontal wavenumber solution given by equation (8) are shown. Horizontal wavenumbers have been chosen empirically to fit the full solution curves; $k_{x}=2.5 / r, k_{y}=1.5 / r$ for the broadside location and $k_{x}=5.5 / r, k_{y}=5.5 / r$ for the end-on position. The gross aspects of the curves are well matched. The example shown is for a $100 \Omega-m$ half-space, and a $500 m$ source receiver separation. For other values of $r$ the two curves shift together, to higher frequency for smaller $r$. For other half-space resistivities the two curves also shift together, to lower frequency and greater apparent resisitivity for larger half-space resistivities.

Figures 1-4 show the basic behavior of a single horizontal wavenumber approximation to CSMT impedance data. The approximation shown there makes no explicit consideration of the air-earth interface. A more rigorous single wavenumber approximation can be made by using a single pair of $k_{x}, k_{y}$ term from a 2-D wavenumber expansion of the full solution for induction by a dipole source. One can write the electric and magnetic fields at wavenuniber $k_{x}$ and $k_{y}$ at the surface of a layered medium as (Ward and Hohman, 1988, equations 4.140, 4.148),

$$
-\frac{8 \pi^{2}}{I d s} E_{x}\left(k_{x}, k_{y}\right)=\frac{u_{0}}{\hat{\sigma}_{0}} \frac{\left(1-r_{T M}\right) k_{x}^{2}}{k_{x}^{2}+k_{y}^{2}}-\frac{i \omega \mu}{u_{0}} \frac{\left(1+r_{T E}\right) k_{y}^{2}}{k_{x}^{2}+k_{y}^{2}}
$$

and

$$
-\frac{8 \pi^{2}}{I d s} H_{y}\left(k_{x}, k_{y}\right)=\frac{\left(1+r_{T M}\right) k_{x}^{2}}{k_{x}^{2}+k_{y}^{2}}+\frac{\left(1-r_{T E}\right) k_{y}^{2}}{k_{x}^{2}+k_{y}^{2}}
$$

where $I d s$ is the electric dipole moment, $\hat{\sigma}_{0}=\sigma_{0}+i \omega \varepsilon_{0}$ is the effective conductivity of the air at frequency $\omega, u_{0}$ is the vertical wavenumber in the air given by

$$
u_{0}^{2}=k_{x}^{2}+k_{y}^{2}-\omega^{2} \mu_{0} \varepsilon_{0}+i \sigma_{0} \omega \mu_{0}
$$

the last two terms of which may be negligible. Coefficients $r_{T M}$ and $r_{T E}$ are wavenumber dependent reflection coefficients which depend on the layered structure, and take into account the matching of field continuity conditions at layer interfaces. On a half-space of 
conductivity $\sigma_{1}$, with $\sigma_{1} \gg \omega \varepsilon_{0}, k_{x}^{2}+k_{y}^{2} \gg \omega \mu_{0} \varepsilon_{0}$, and $\sigma_{0}=0$, reflection coefficients given by Ward and Hohman (1988) are such that the impedance at wavenumber $k_{x}, k_{y}$ reduces to

$$
\frac{E_{x}}{H_{y}}=\frac{u_{1} / \hat{\sigma}_{1} k_{x}^{2}-i \omega \mu_{0} /\left(u_{0}+u_{1}\right) k_{y}^{2}}{k_{x}^{2}-u_{1} /\left(u_{0}+u_{1}\right) k_{y}^{2}} .
$$

Apparent resistivity and phase are plotted for receivers in the broadside (Figures 5 and 6) and end-on (Figures 7 and 8 ) locations. Both values calculated from the full solution for induction in a half-space by a horizontal electric dipole and for single horizontal wavenumber solution (12) are shown. Horizontal wavenumbers have been chosen empirically to fit the full solution curves; $k_{x}=0.9 / r, k_{y}=0.8 / r$ for the broadside location and $k_{x}=1.6 / r, k_{y}=1.5 / r$ for the end-on position. Comparing with the figures for the previous approximation, the fit to the broadside response is considerably improved. The fit to the end-on response is less good.

One might seek to improve the fit of a single wave number approximation by moving from a 2-D Fourier domain to a Hankell transform domain. In effect, this integrates the electric and magnetic fields around rings of constant $\lambda^{2}=k_{x}^{2}+k_{y}^{2}$. A single Hankel wavenumber $\lambda$ corresponds to a ring of Fourier wavenumbers $k_{x}, k_{y}$. We have tried this approach and found that it improves the end-on receiver response fit and degrades the fit to the broadside receiver response (not shown). The fit of this approximation to the broadside response can be improved by approximating the full solution with fields summed over a pair of Hankel wavenumbers resulting in curves very similar to Figures 5 and 6 (not shown).

\section{Conclusion and suggestion for future work}

Initial results on finite wavenumber effects in CSMT are encouraging in that very simple considerations of the necessarily finite source wavenumbers give a good approximation to the gross aspects of both broadside and end-on data. A very exciting aspect of the approximation is that the appropriate wavenumbers appear to depend only on source-receiver separation and orientation. This implies that a correction to CSMT data can be made based solely on source-receiver configurations. With the current single wavenumber approximations, the results will evidently be more accurate for the broadside receiver position, as the approximation is more accurate in that case. We hope that the accuracy will be substantially improved by introducing a second term encompassing integrated effects of the $k_{x}, k_{y} \rightarrow \infty$ asymptotes. Evidently, further work is needed to refine the corrections and investigate their ranges of validity.

An interesting and potentially very useful outcome of this research is that impedance measurements at low frequencies may be useful by themselves. This observation is from the conclusion that there is a single pair of wavenumbers $\left(k_{x}, k_{y}\right)$ that can approximately represent the full EM impedance at low frequencies. In principle, we can recover the resistivity structure of the deeper portion of a layered earth by inverting 
the impedance data for the pair of wavenumbers and the layered-earth resistivity together. The inversion is in the wavenumber domain in which the impedance is in a closed form. The efficiency in speed and the minimum memory requirement involved in the wavenumber-domain inversion will allow us real-time operation on a PC platform.

\section{Acknowledgment}

This work was supported by the Assistant Secretary for Energy Efficiency and Renewable Energy, Geothermal Division of the U.S. Department of Energy under Contract No. DE-AC03-76SF00098.

\section{Reference}

Newman, G.A., 1995, Crosswell electromagnetic inversion using integral and differential equations, Geophysics, 59, 371-935.

Ward, S.H., and Hohmann, G.W., 1988. Electromagnetic theory for geophysical applications, in, M.W. Nabighian, Ed., Electromagnetic Methods in Applied Geophysics Theory: vol. 1, Society for Exploration Geophysicists, Tulsa.

Xie, G., and Lee, K.H., 1995, Nonlinear inversion of 3-D electromagnetic data, Proc. On Progress in Electromagnetic Research Symp., Univ. of Washington, Seatle, USA. 


\section{List of figures}

Figure 1. Apparent resistivity $\rho_{x y}$ as a function of frequency, for a $J_{x}$ dipole source on a $100 \Omega-m$ half space, and for single wavenumber approximation (8). Receiver is 500 $m$ from source in a broadside position. Wavenumbers $k_{x}=2.5 / r$ and $k_{y}=1.5 / r$ have been used in approximation (8).

Figure 2. Impedance phase $\theta_{x y}$ as a function of frequency, for a $J_{x}$ dipole source on a 100 $\Omega-m$ half space, and for single wavenumber approximation (8). Receiver is $500 \mathrm{~m}$ from source in a broadside position. Wavenumbers $k_{x}=2.5 / r$ and $k_{y}=1.5 / r$ have been used in approximation (8).

Figure 3. Apparent resistivity $\rho_{x y}$ as a function of frequency, for a $J_{x}$ dipole source on a $100 \Omega-m$ half space, and for single wavenumber approximation (8). Receiver is 500 $m$ from source in an end-on position. Wavenumbers $k_{x}=5.5 / r$ and $k_{y}=5.5 / r$ have been used in approximation (8).

Figure 4. Impedance phase $\theta_{x y}$ as a function of frequency, for a $J_{x}$ dipole source on a 100 $\Omega-m$ half space, and for single wavenumber approximation (8). Receiver is $500 \mathrm{~m}$ from source in an end-on position. Wavenumbers $k_{x}=5.5 / r$ and $k_{y}=5.5 / r$ have been used in approximation (8).

Figure 5. Apparent resistivity $\rho_{x y}$ as a function of frequency, for a $J_{x}$ dipole source on a $100 \Omega-m$ half space, and for single wavenumber approximation (12). Receiver is $500 \mathrm{~m}$ from source in a broadside position. Wavenumbers $k_{x}=0.9 / r$ and $k_{y}=0.8 / r$ have been used in approximation (12).

Figure 6. Impedance phase $\theta_{x y}$ as a function of frequency, for a $J_{x}$ dipole source on a 100 $\Omega-m$ half space, and for single wavenumber approximation (12). Receiver is $500 \mathrm{~m}$ from source in a broadside position. Wavenumbers $k_{x}=0.9 / r$ and $k_{y}=0.8 / r$ have been used in approximation (12).

Figure 7. Apparent resistivity $\rho_{x y}$ as a function of frequency, for a $J_{x}$ dipole source on a $100 \Omega-m$ half space, and for single wavenumber approximation (12). Receiver is $500 \mathrm{~m}$ from source in an end-on position. Wavenumbers $k_{x}=1.6 / r$ and $k_{y}=1.5 / r$ have been used in approximation (12).

Figure 8. Impedance phase $\theta_{x y}$ as a function of frequency, for a $J_{x}$ dipole source on a 100 $\Omega-m$ half space, and for single wavenumber approximation (12). Receiver is $500 \mathrm{~m}$ from source in an end-on position. Wavenumbers $k_{x}=1.6 / r$ and $k_{y}=1.5 / r$ have been used in approximation (12). 


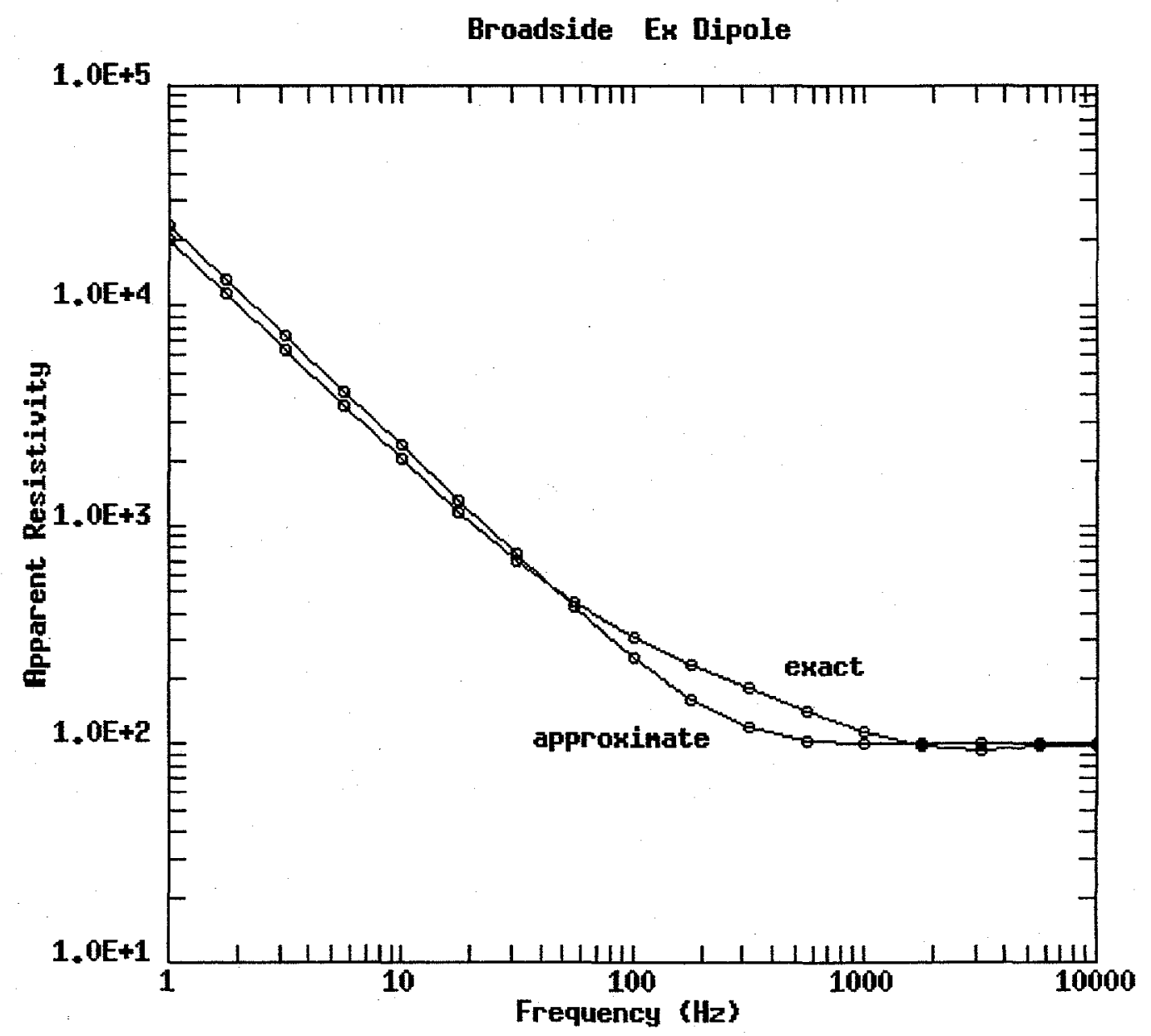

Figure 1. Apparent resistivity $\rho_{x y}$ as a function of frequency, for a $J_{x}$ dipole source on a $100 \Omega-m$ half space, and for single wavenumber approximation (8). Receiver is $500 \mathrm{~m}$ from source in a broadside position. Wavenumbers $k_{x}=2.5 / r$ and $k_{y}=1.5 / r$ have been used in approximation (8). 


\section{Broadside Ex Dipole}

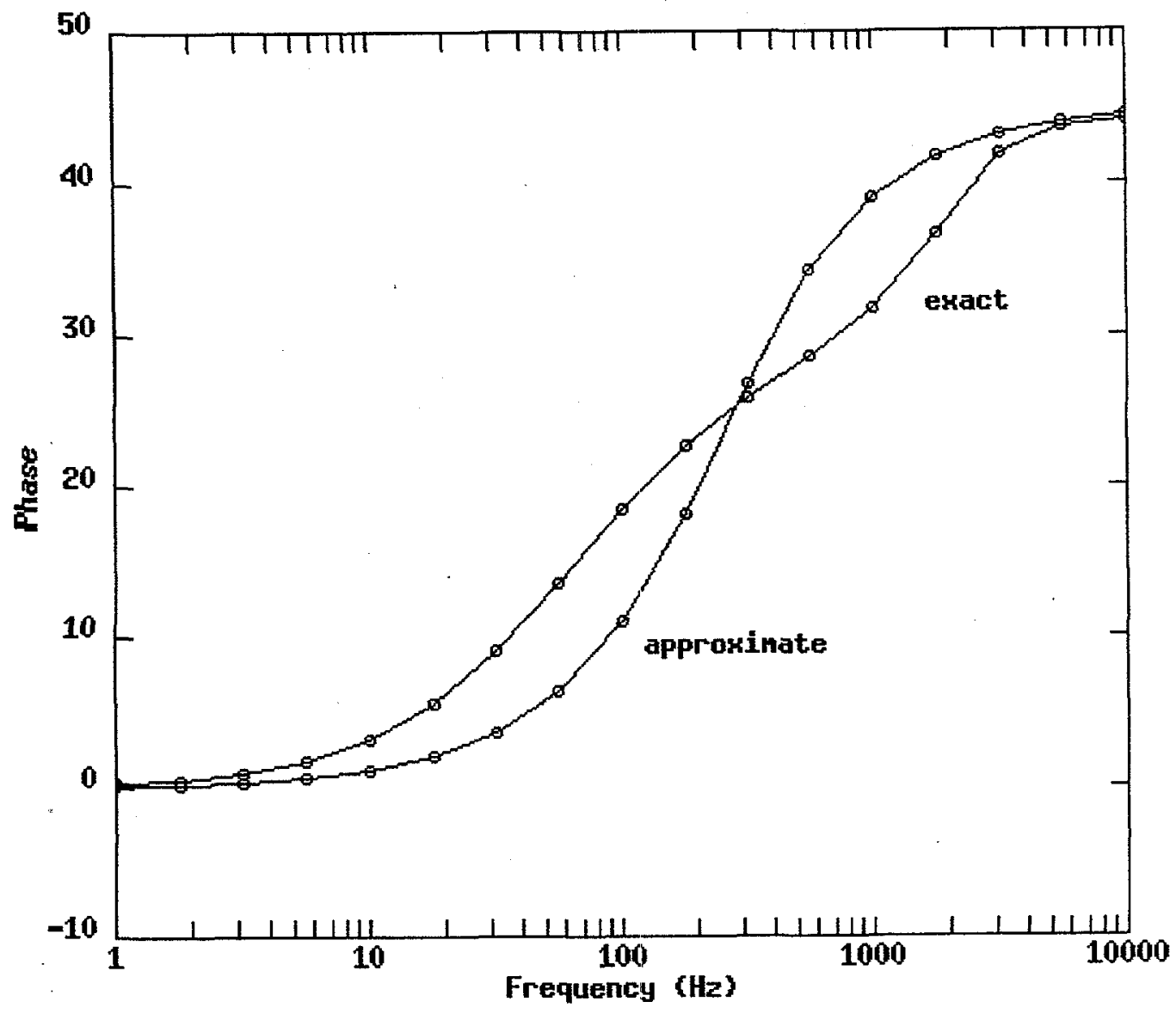

Figure 2. Impedance phase $\theta_{x y}$ as a function of frequency, for a $J_{x}$ dipole source on a $100 \Omega-m$ half space, and for single wavenumber approximation (8). Receiver is $500 \mathrm{~m}$ from source in a broadside position. Wavenumbers $k_{x}=2.5 / r$ and $k_{y}=1.5 / r$ have been used in approximation (8). 


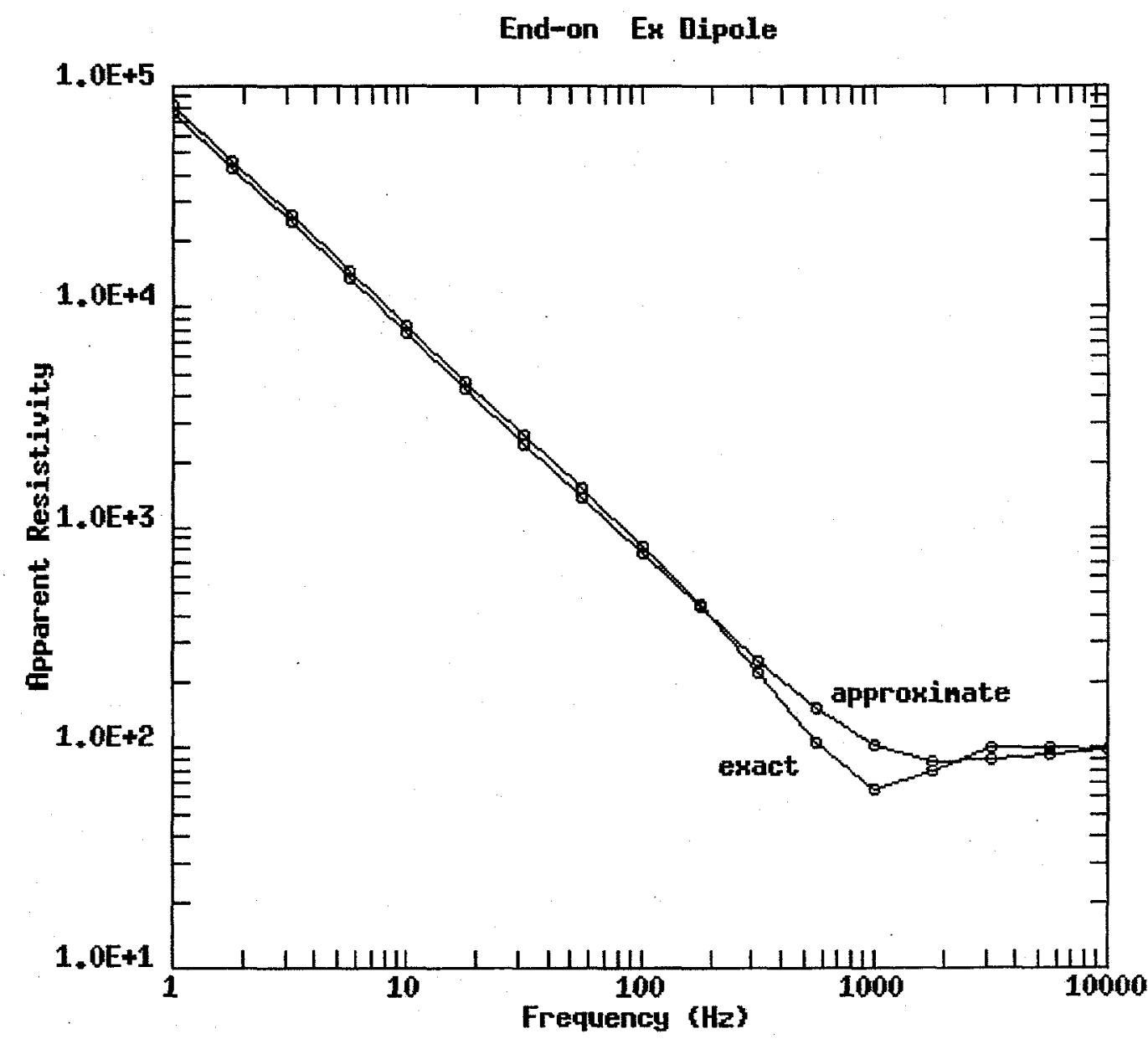

Figure 3. Apparent resistivity $\rho_{x y}$ as a function of frequency, for a $J_{x}$ dipole source on a $100 \Omega-m$ half space, and for single wavenumber approximation (8). Receiver is $500 m$ from source in an end-on position. Wavenumbers $k_{x}=5.5 / r$ and $k_{y}=5.5 / r$ have been used in approximation (8). 


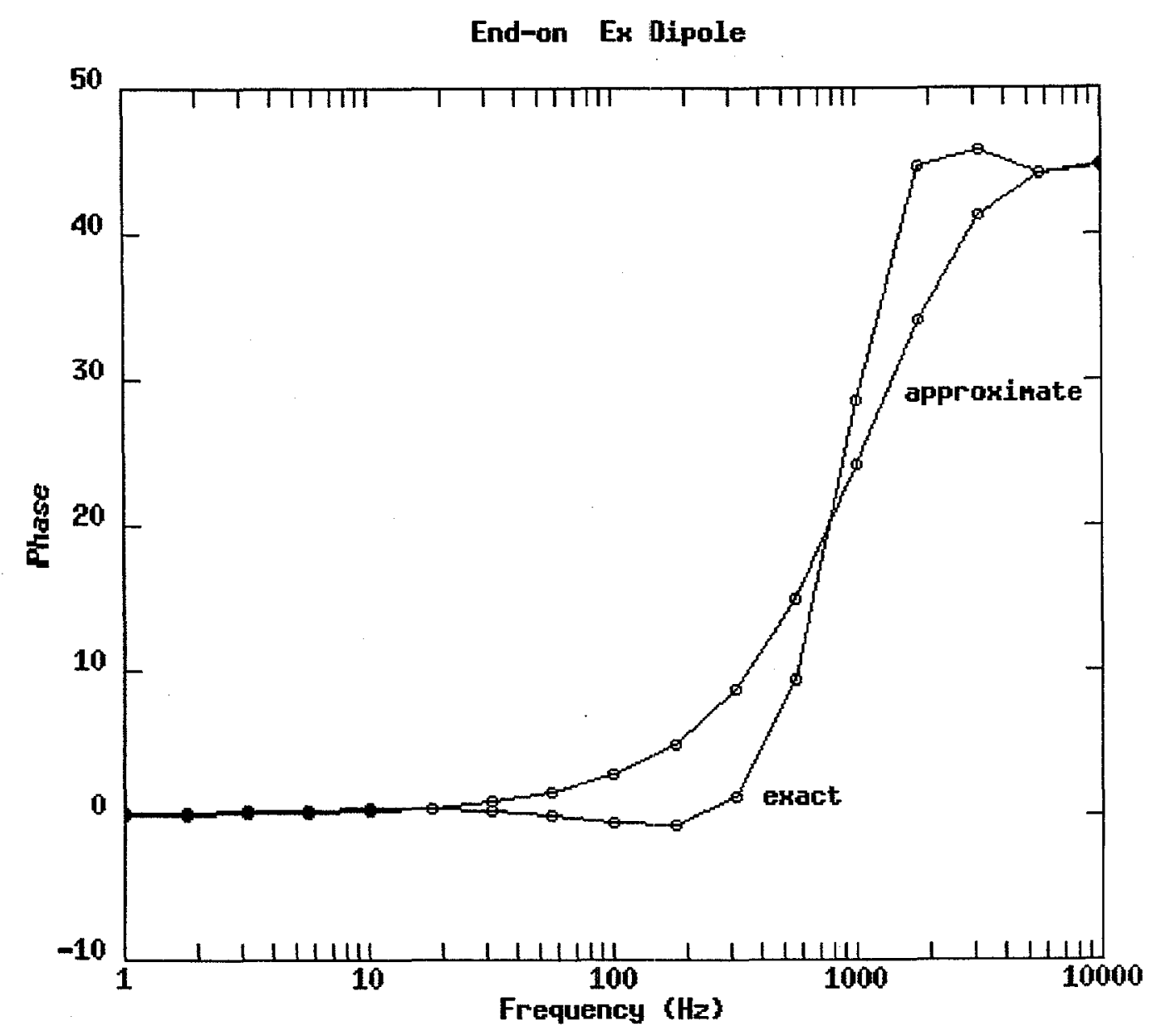

Figure 4. Impedance phase $\theta_{x y}$ as a function of frequency, for a $J_{x}$ dipole source on a $100 \Omega-m$ half space, and for single wavenumber approximation (8). Receiver is $500 m$ from source in an end-on position. Wavenumbers $k_{x}=5.5 / r$ and $k_{y}=5.5 / r$ have been used in approximation (8). 


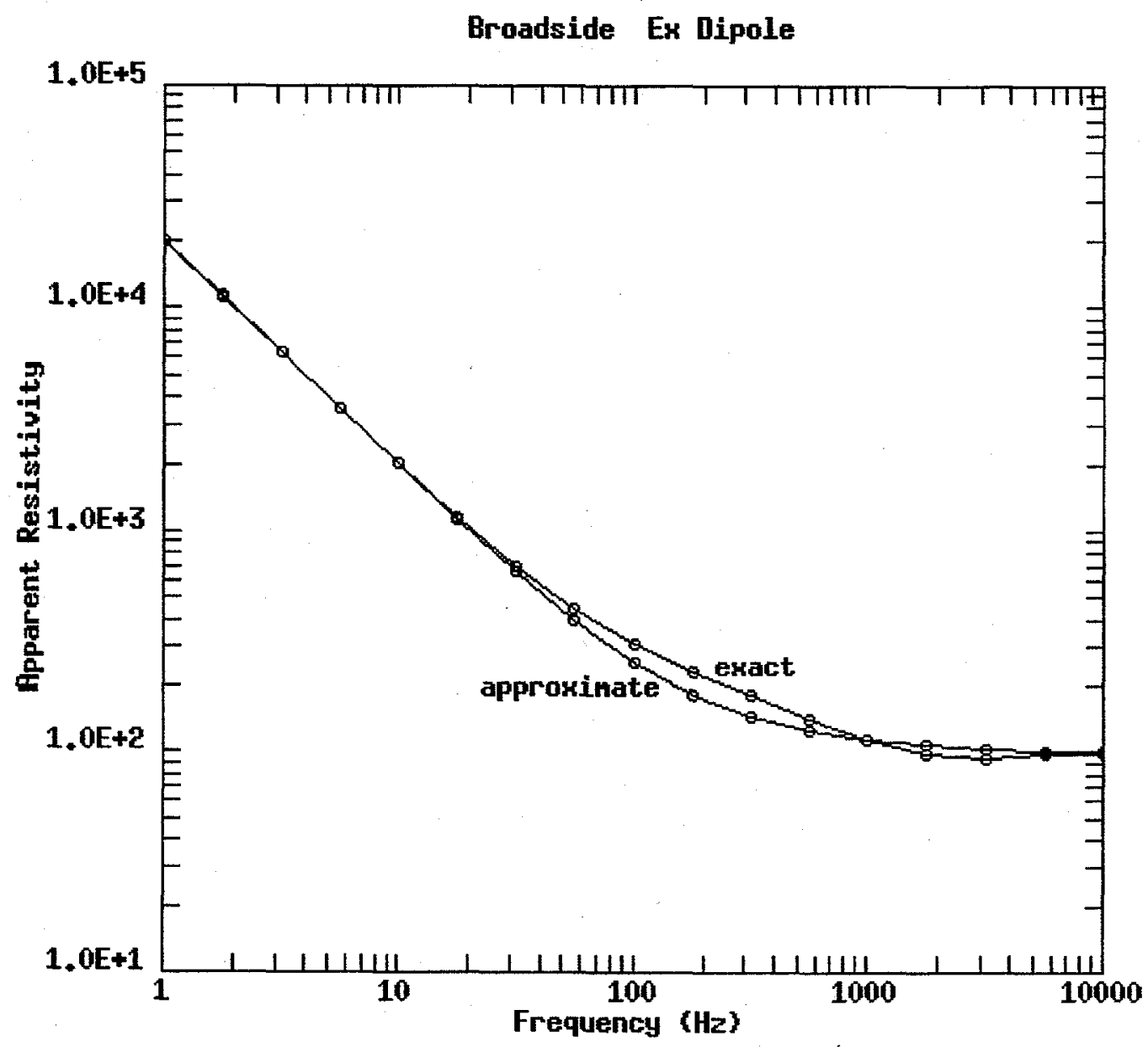

Figure 5. Apparent resistivity $\rho_{x y}$ as a function of frequency, for a $J_{x}$ dipole source on a $100 \Omega-m$ half space, and for single wavenumber approximation (12). Receiver is $500 m$ from source in a broadside position. Wavenumbers $k_{x}=0.9 / r$ and $k_{y}=0.8 / r$ have been used in approximation (12). 


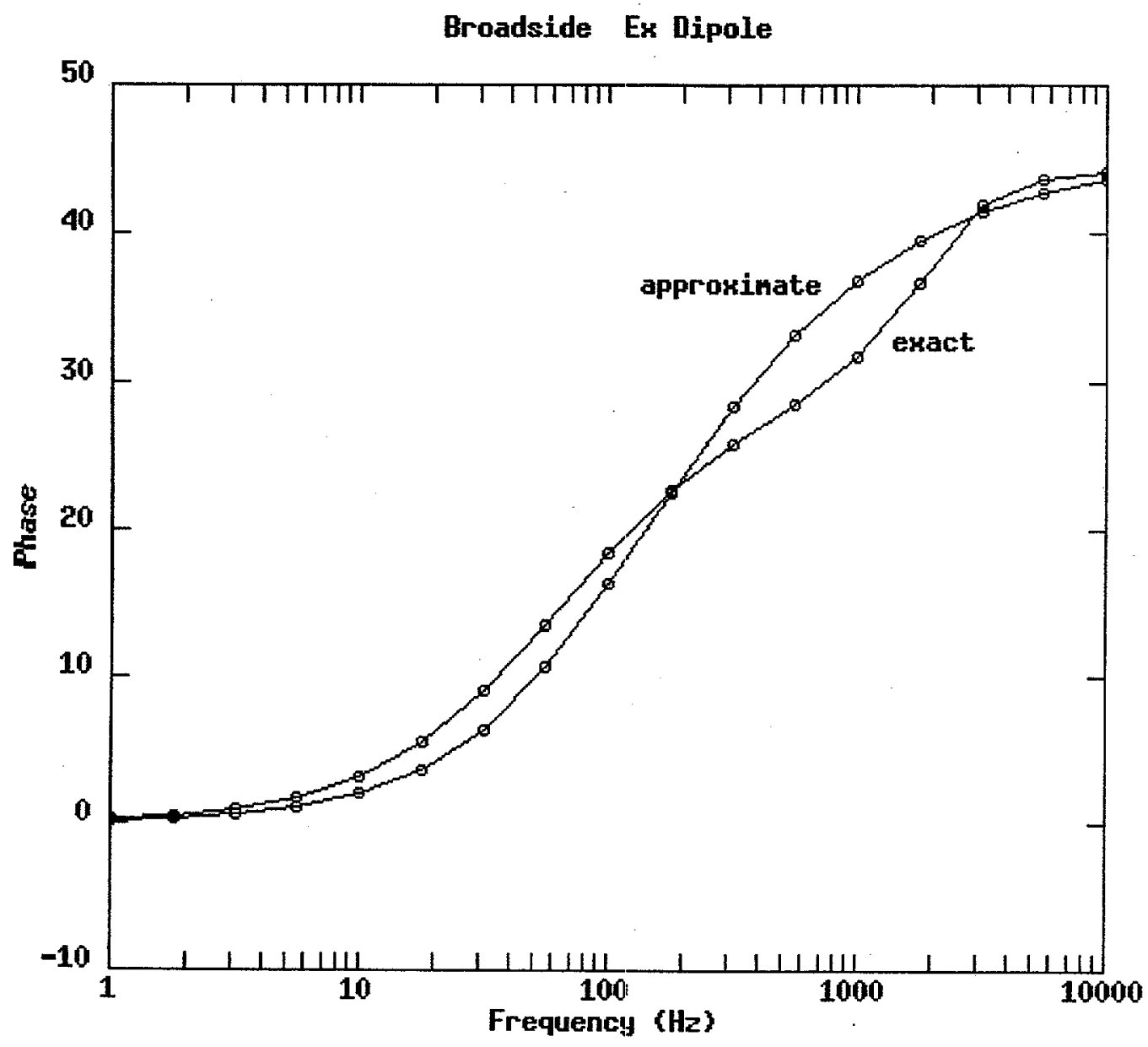

Figure 6. Impedance phase $G_{x y}$ as a function of frequency, for a $J_{x}$ dipole source on a $100 \Omega-m$ half space, and for single wavenumber approximation (12). Receiver is $500 \mathrm{~m}$ from source in a broadside position. Wavenumbers $k_{x}=0.9 / r$ and $k_{y}=0.8 / r$ have been used in approximation (12). 


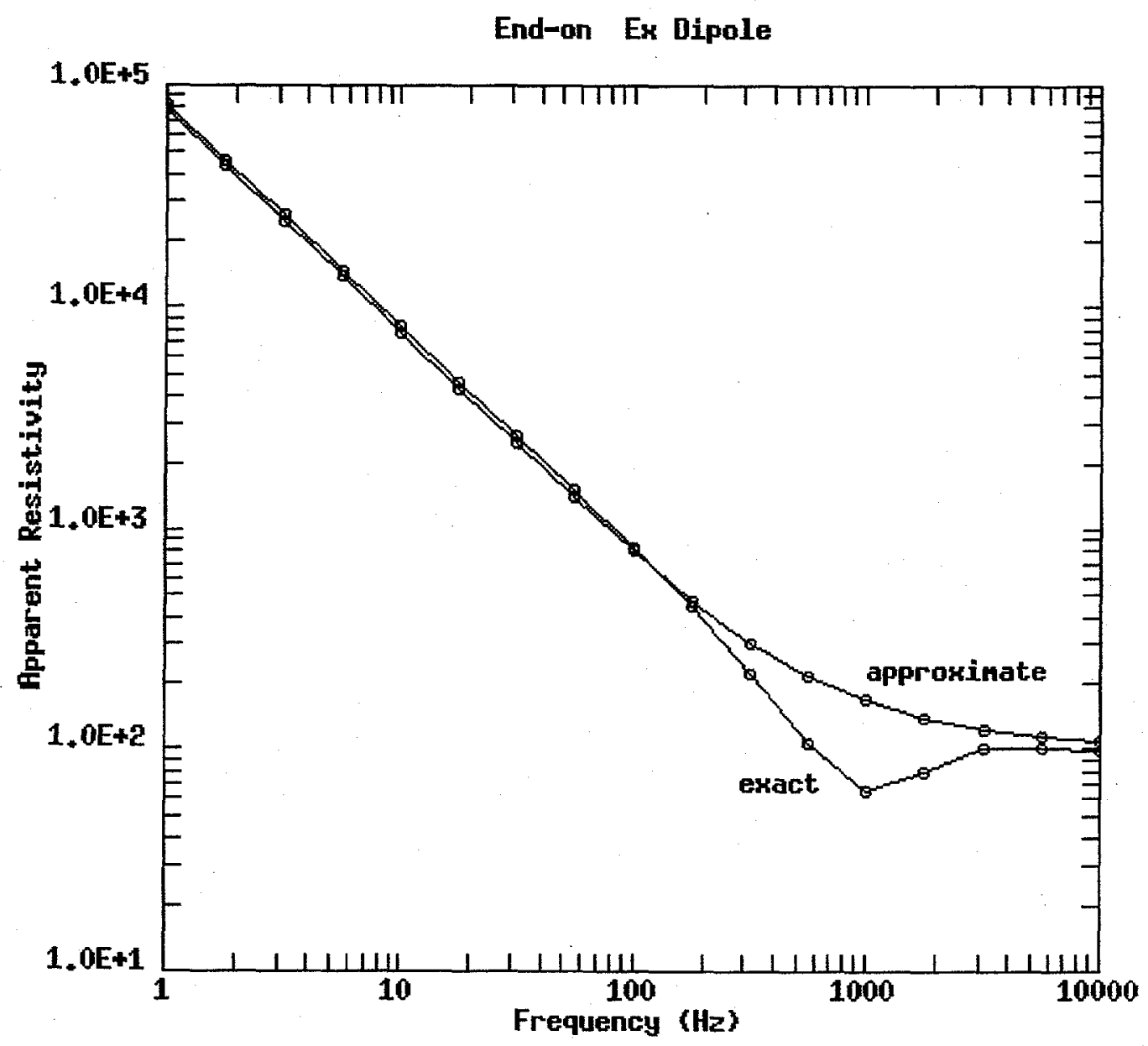

Figure 7. Apparent resistivity $\rho_{x y}$ as a function of frequency, for a $J_{x}$ dipole source on a $100 \Omega-m$ half space, and for single wavenumber approximation (12). Receiver is $500 \mathrm{~m}$ from source in an end-on position. Wavenumbers $k_{x}=1.6 / r$ and $k_{y}=1.5 / r$ have been used in approximation (12). 


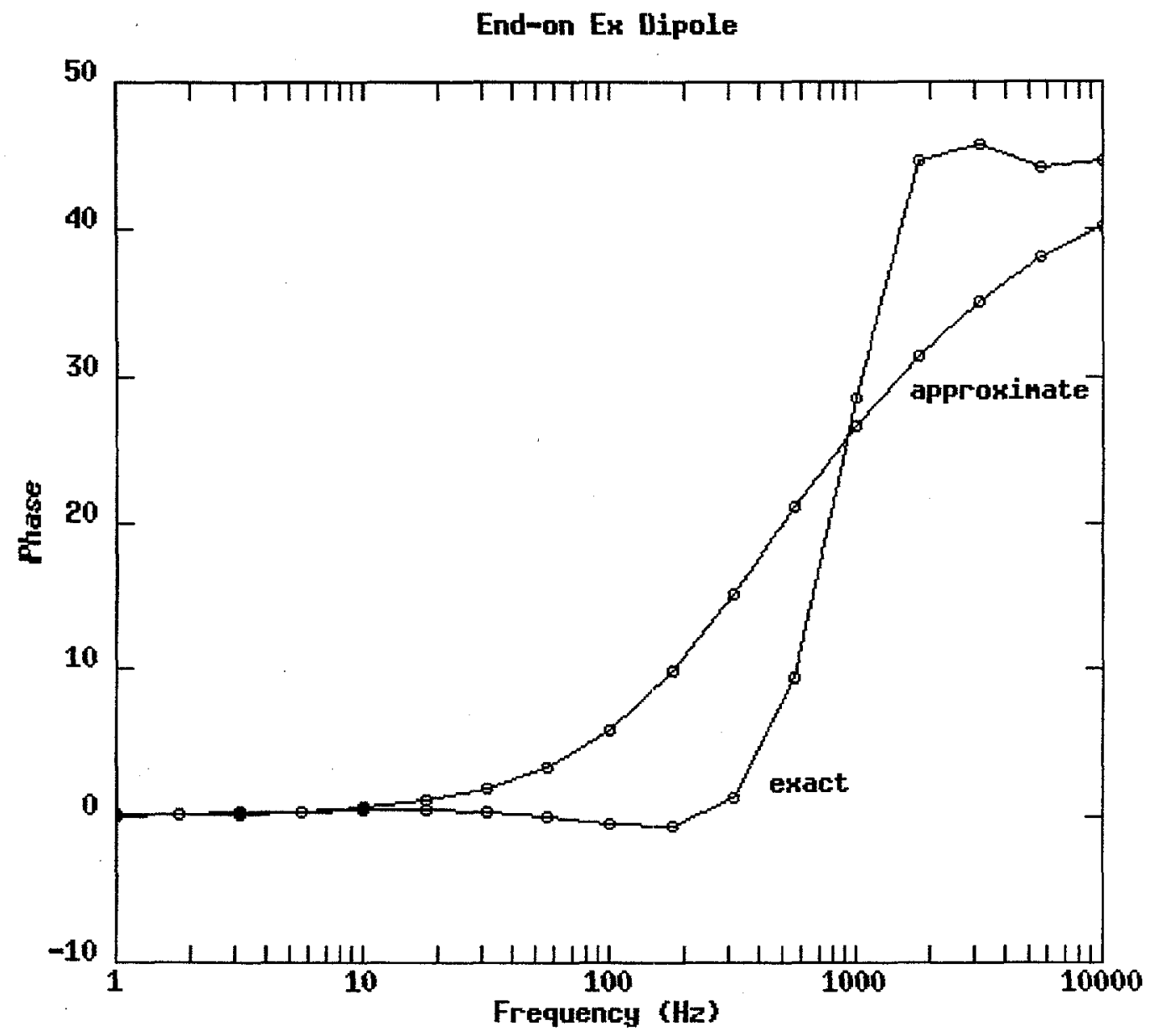

Figure 8. Impedance phase $\theta_{x y}$ as a function of frequency, for a $J_{x}$ dipole source on a $100 \Omega-m$ half space, and for single wavenumber approximation (12). Receiver is $500 \mathrm{~m}$ from source in an end-on position. Wavenumbers $k_{x}=1.6 / r$ and $k_{y}=15 / r$ have been used in approximation (12). 